\title{
EDIFİCATE
}

I Congreso de Escuelas de Edificación y Arquitectura Técnica de España

València, 4 y 5 de noviembre de 2021

Escuela Técnica Superior de Ingeniería de Edificación

Universitat Politècnica de València

Doi: https://doi.org/10.4995/EDIFICATE2021.2021.13608

\section{La inserción de las asignaturas de carácter legal en la titulación de arquitecto técnico}

\section{The insertion of legal subjects in the degree of technical architect}

\author{
$M^{\mathbf{a}}$ Jesús Romero Aloy \\ Profesora Titular de Universidad. Área de Derecho Administrativo del Departamento de Urbanismo. \\ Universitat Politècnica de Valencia, maroal1@urb.upv.es
}

\begin{abstract}
This communication presents the teaching experience during the last 20 years in the subjects of legal content taught at the Higher Technical School of Building Engineering of the Polytechnic University of Valencia. Teaching methodologies are proposed that, starting from the lecture, must necessarily give way to active methodologies based on guided practices, group work by students and the development of projects based on the knowledge acquired.
\end{abstract}

Keywords: teaching methodologies, legislation, urban management, projects.

\section{Resumen}

Esta comunicación presenta la experiencia docente durante los últimos 20 años en las asignaturas de contenido legal impartidas en la Escuela Técnica Superior de Ingeniería de la Edificación de la Universidad Politécnica de Valencia. Se proponen metodologías docentes que, partiendo de la lección magistral, necesariamente deben dar paso a metodologías activas basadas en las prácticas guiadas, los trabajos grupales de los alumnos y el desarrollo de proyectos basados en los conocimientos adquiridos.

Palabras clave: metodologías docentes, legislación, gestión urbanística, proyectos. 


\section{Introducción: la actitud inicial del alumnado y finalidad de esta comunicación}

En primer lugar, he de referirme a la actitud del alumnado ante las asignaturas de contenido legal en las escuelas técnicas, compartiendo mi experiencia adquirida a lo largo de veintidós años de enseñanza. En principio, puede decirse que el alumno acusa una cierta sorpresa al observar que en el plan de estudios tiene una asignatura llamada Legislación y otra denominada Gestión Urbanística, cursadas en segundo, cuyo contenido es eminentemente legal.

Esa desubicación inicial está en proporción a la madurez del alumnado. Así, se comprueba que en los grupos de la tarde a los que suelen acudir alumnos de mayor edad y con mayor experiencia que en los grupos de la mañana, aquéllos tienen una actitud de mayor aprecio y de positiva valoración que en los grupos de la mañana en los que el alumnado es generalmente de menor madurez.

En cualquier caso, a medida que se avanza en las explicaciones de las asignaturas, se observa un progresivo interés en la medida que el alumno capta la relación de la materia impartida con la vida real en la que se inserta el trabajo profesional.

Por tanto, el propósito de esta comunicación es poder hacer comprender al alumno desde el primer momento la necesidad que tiene de conocer bien el contenido de estas asignaturas, ya que de lo contrario el ejercicio profesional se verá afectado negativamente por fuertes limitaciones. Si esto se asimila desde el inicio de la asignatura, el alumno cursará las asignaturas desde una perspectiva mucho más favorable. Por el contrario, si mantiene una cierta nebulosa sobre la utilidad de lo que tiene que aprender, sin que se desvanezca su extrañeza sorpresa inicial evidentemente su actitud será de menor motivación e interés.

Este cometido que nos proponemos no es necesario en las otras materias que componen el plan de estudios de la carrera en las que el alumno no necesita de ninguna aclaración para comprender que debe conocerlas como puede ser el caso de la construcción o de las instalaciones.

\section{Visión histórica: el tronco común, la Arquitectura Legal}

Por el impacto social que produce la edificación, ha estado sometida a normas desde tiempos remotos. Algunas de las determinaciones hoy vigentes en el Código Civil sobre servidumbres y otras limitaciones de la propiedad tienen su origen en el Derecho Romano. Por referirnos a épocas menos antiguas, es significativo el Acuerdo que en el año 1759 adoptó la Real Academia de San Fernando ante el desorden que reinaba en las enseñanzas de la arquitectura, acordó nombrar una comisión que elaborara un plan de estudios, propuesta que después de diversas vicisitudes, cristalizó en la Real Orden de 8 de enero de 1850, por la que se formalizó el plan de estudios de la titulación de arquitecto donde, en el cuarto curso de la carrera, ya aparecía la asignatura de Arquitectura Legal, en 
la que se encuentra el núcleo de los contenidos legales que, desde hace casi dos siglos se vienen impartiendo en la titulaciones universitarias.

Desde el punto de vista académico, tempranamente aparecen tratados importantes que desarrollan las asignaturas de contenido legal. El primero de ellos es el Tratado de la Legislación de Arquitectura, Agrimensura y Caminos Vecinales de Marcial de la Cámara. En 1870, aparece el Tratado de Arquitectura Legal de Calvo y Pereira. Pocos años después de la publicación del Código Civil, se publica la obra de Martínez Ángel y Oyuelos Pérez, también titulado Tratado de Arquitectura Legal, obra muy sistemática ya que aprovecha el tratamiento que el Código Civil efectúa de las materias afectantes a la edificación.

Como puede comprobarse, a partir del momento en el que aparece publicado el primer plan de estudios de la titulación de Arquitecto, comienzan a publicarse importantes tratados sobre arquitectura legal que constituyen la base científica de la materia. Así pues en el siglo XIX, ya existe un importante cuerpo doctrinal que sirve de pilar para la enseñanza de estos contenidos.

\section{La relación de las asignaturas Legislación y Gestión Urbanística con las atribuciones profesionales}

Estas asignaturas sirven directamente a un importante grupo de competencias profesionales que, de no ser por estas disciplinas, el titulado saldría de la escuela siendo incapaz de atender tales trabajos. El temario impartido parte del análisis de las competencias profesionales del arquitecto técnico y sus correspondientes necesidades formativas en la materia.

La gran parte de atribuciones profesionales del arquitecto técnico se encuentran reguladas en la Ley 38/1999, de Ordenación de la Edificación y en la Ley 12/1986, de atribuciones profesionales de Ingenieros y Arquitectos Técnicos.

Concretamente, las competencias profesionales de los arquitectos técnicos se regulan en el Real Decreto 927/1992, de 17 de julio, por el que se establece el título universitario oficial de Arquitecto Técnico y la aprobación de las directrices generales propias de los planes de estudios conducentes a la obtención de aquél, y en la Orden ECl 3855/2007, de 27 de diciembre, por la que se establecen los requisitos para la verificación de los títulos universitarios oficiales que habiliten para el ejercicio de la profesión de Arquitecto Técnico.

Acudiendo a la citada Ley 12/1986, de 1 de abril, reguladora de las atribuciones de los arquitectos técnicos, podemos identificar aquellos trabajos profesionales para cuyo ejercicio resulta indispensable el contenido de las asignaturas que estamos tratando. Un superficial análisis nos lo demuestra:

1. Las atribuciones profesionales dentro del ámbito de los proyectos de intervención en la edificación necesitan del conocimiento de los planes de ordenación en los que figuran las determinaciones urbanísticas a las cuales el 
proyecto debe someterse. Si estas determinaciones se desconocen o se interpretan de modo erróneo, la intervención en el edificio no podrá realizarse. En las asignaturas se presta una considerable atención al conocimiento de las distintas figuras de planeamiento.

2. La gestión administrativa de las licencias de obra: todas las posibles intervenciones de edificación para poder realizarse, necesitan de la correspondiente licencia de obra. Si ésta no existe no se puede intervenir. El arquitecto técnico necesita conocer bien el contenido y estructura de la licencia de obra, no sólo para confeccionar la documentación correctamente, sino para ser capaz de intervenir como parte ante el ayuntamiento o en el proceso de tramitación, en el cual muchas veces es preciso subsanar deficiencias o aportar nuevos datos.

3. Como principal responsable de la técnica constructiva el profesional debe conocer lo que es un contrato de obra, ya que muchas veces será el responsable del seguimiento y correcta ejecución del mismo. Es el denominado proyect management o gestión del proyecto.

4. Las valoraciones inmobiliarias en las que el arquitecto técnico tiene cada vez un mayor protagonismo. Para valorar un solar o una parcela, es preciso conocer las determinaciones urbanísticas de las que depende su valor tales como la edificabilidad, aprovechamiento tipo, cesiones de suelo a realizar, obras pendientes de urbanización a cargo del propietario, previsiones temporales de gestión urbanística, etc. Por otra parte, es imprescindible conocer la calificación urbanística de suelo urbano, urbanizable o no urbanizable. Sin el conocimiento de estos elementos derivados del planeamiento y de la gestión urbanística es imposible realizar la valoración de un solar o de una parcela.

5. La responsabilidad profesional en el ejercicio de la profesión. En la práctica profesional del arquitecto técnico se depositan unos intereses económicos, en ocasiones importantes en cuantía y, respecto de su tarea como director de ejecución de obra, se le requerirá un resultado adecuado, por lo que el ejercicio de la profesión genera un importante impacto social cuyas responsabilidades civiles, penales y administrativas, tienen una gran importancia. Por tanto, resulta necesario que el alumno conozca las responsabilidades en las que puede incurrir durante el ejercicio de la profesión, ya sean civiles, penales, o meramente administrativas.

6. La tramitación de expedientes en las licencias ambientales (de apertura) de todo tipo de establecimientos. El alumno recibe la formación adecuada sobre los instrumentos de control administrativo ambiental que siguen la fiscalización de aquellas instalaciones y actividades susceptibles de producir molestias, alterar las condiciones de salubridad del medio ambiente u ocasionar daños o riesgos a las personas o el medio ambiente. 
7. La preparación que suponen estas asignaturas para quienes vayan a optar a algún puesto en la administración pública en los diferentes niveles de administración estatal, autonómica o municipal. Los temarios de estas oposiciones tienen una importante parte de materias legales (oposiciones para nombramientos interinos, bolsas de trabajo ...) por lo que el conocimiento de las materias legales a las que nos estamos refiriendo es imprescindible.

8. Todas las servidumbres que afectan a la edificación tales como las servidumbres de vistas, de luces, de paso, de medianería, etc. Es de capital importancia el conocimiento de estas limitaciones de la propiedad ya que, de no observarlas, el técnico incurre en la correspondiente responsabilidad.

9. Toda la normativa referente a las viviendas de protección pública en cuyos expedientes el arquitecto técnico tiene plena competencia para su tramitación y para la realización de determinadas intervenciones en este tipo de viviendas.

10. El conocimiento de las medidas de defensa a su alcance para defenderse ante posibles perjuicios en algún inmueble como son los juicios verbales de obra nueva o los de obra ruinosa.

\section{La visión de la panorámica global en la que se inserta la edificación y la asignatura de Gestión Urbanística}

Esta asignatura proporciona al estudiante una nueva visión de la edificación, ya que aparece insertada dentro de un complejo instrumento cual es el plan de ordenación, que contiene las alineaciones, los posibles usos del edificio, las alturas edificables, la tipología edificatoria, los condicionantes estéticos, etc..

El impacto de esta asignatura sobre la visión de la edificación y de la arquitectura es muy importante, ya que se le ofrece al alumno no la visión particular e individualizada, sino una visión total desde la cual se comprende y se justifica la edificación en cada caso particular.

La posible pieza se inserta, además, sobre un suelo que tiene una determinada clasificación urbanística de urbano, urbanizable o no urbanizable, lo cual supone que pueda edificarse en un breve periodo de tiempo, como es el caso del suelo urbano, tras la obtención de la correspondiente licencia de edificación, o bien que para poder edificarse haya de seguirse un largo periodo de gestión que puede durar años, después del cual el suelo contará con los correspondientes servicios urbanísticos, como es el caso del suelo urbanizable. O bien que no pueda edificarse en absoluto o sólo en situaciones muy particulares, como es el caso del suelo no urbanizable.

La construcción de la ciudad pasa inevitablemente por complejos procesos de gestión urbanística como resultado de los cuales se obtiene la urbanización de los viarios, abastecimiento de agua, desagües, alumbrado público y obtención de suelos dotacionales para zonas verdes, centros educativos, asistenciales, etc. Todo ello supone importantes desembolsos por parte de los propietarios para el pago de las obras de urbanización que, 
además, deben ceder gratuitamente la parte proporcional que les corresponde de suelo para viarios y dotaciones.

Esta compleja operación debe realizarse según unos criterios de reparto proporcional que exige la formulación de conceptos como son los de aprovechamiento tipo, coeficientes de homogenización, etc. Como resultado de los cuales se obtiene el aprovechamiento urbanístico que en cada caso corresponde al propietario.

Estos conceptos definen el valor del suelo y en consecuencia son de imprescindible conocimiento a la hora de que el profesional realice la valoración de un determinado suelo.

En este sentido, y con carácter global, cabe decir que el urbanismo es una ciencia multidisciplinar $^{1}$ que admite la intervención de numerosas ramas profesionales, lógicamente, todo ello, en función de las competencias que pueda ejercer cada titulación y, el arquitecto técnico goza de las competencias profesionales para ejercer su profesión en materia de urbanismo, ya sea dentro del ejercicio libre de la profesión o en su trabajo desde la administración pública dentro de los servicios técnicos de urbanismo. Pues bien, esta asignatura le otorga los conocimientos necesarios para que, en su ejercicio profesional, puedan desarollar exitosamente las labores en materia de urbanismo tales como: proyectos de reparcelación, segregación y agrupación de parcelas, instrumentos de planeamiento, proyectos de urbanización, expedientes de legalización de infracciones urbanísticas, etc. Dentro de los servicios técnicos de urbanismo en la administración, tendrán competencias para realizar informes técnicos urbanísticos en los expedientes de licencias de obras, informes para laconcesión de licencias de agrupación, segregación, reparcelación o modificación de linderos, informes de situación urbanística, informes de compatibilidad urbanística, apertura de expedientes de infracciones urbanísticas, etc

\section{La metodología docente}

Debemos partir de la idea de cambio del sistema de "enseñar" por el de "ayudar a aprender", donde los alumnos tengan una actitud activa en su tarea de conocer la materia propuesta (Santana, 2020) ${ }^{2}$, pero tal afirmación no se realiza en desdeño de la clase magistral, puesto que el docente debe enseñar el contenido de la asignatura. Quizás, el cambio con respecto a la clásica lección magistral es que, en las asignaturas de contenido legal, estas explicaciones se realizan desde un punto de vista eminentemente práctico

\footnotetext{
${ }^{1}$ GARCÍA ERVITI, F. et all ( 2013) "Herramientas de aprendizaje sobre regulación legal del ejercicio profesional del arquitecto". En XI Jornadas de Redes de Investigación en Docencia Universitaria. Alicante: Universidad de Alicante, 2659 p. "Las materias propias de la disciplina académica de Arquitectura Legal tienen un carácter pluridisciplinar y transversal en el contexto de las competencias que deben adquirirse para la obtención de los títulos universitarios oficiales que habiliten para el ejercicio de la profesión de Arquitecto, participando tanto del área técnica -con las disciplinas propias de la construcción, estructuras e instalaciones-, como del ámbito proyectual, que incorpora las materias de composición, proyectos y urbanismo”. 2273.

2 SANTANA RODRÍGUEZ, R.J., (2020). "El aprendizaje basado en la práctica profesional : metodologías activas". En Arquitectura v2020. Valencia: Universitat Politècnica de València. 56.
} 
enfocándolas a la práctica constructiva que les permitirá reflejar en la vida real los conceptos aprendidos.

En nuestras asignaturas, Legislación y Gestión Urbanística, se lleva a cabo una docencia presencial mediante clases magistrales; se plantean casos concretos y reales para ilustrar los contenidos teóricos y, se alternan con debates surgidos a partir de las preguntas y comentarios de los alumnos en relación al caso estudiado.

También se desarrollan talleres de trabajos en grupo sobre un caso práctico propuesto y tutelado por el profesor, con el análisis, exposición y discusión de los casos realizados por los alumnos, con una presentación oral de los trabajos con debate posterior y preguntas del resto de compañeros.

En concreto, la metodología docente se desarrolla del siguiente modo:

\subsection{Las clases teóricas}

Es muy importante conseguir una metodología docente que se corresponda con el perfil del alumnado que tiene una formación técnica. Por ello hay que acudir a una metodología con importantes dosis de aporte práctico que sirva para fijar y comprender los conceptos y determinaciones que vienen establecidos en las normas.

Al alumno puede resultarle ininteligible una formulación que se contiene en un determinado precepto legal. A modo de ejemplo, puede pensarse en la regulación de lo que es una actuación integrada o una actuación aislada. La expresión contenida en la norma hay que verla reflejada concretamente sobre un plano de cualquier ejemplo real. Entonces, el alumno pasa de una nebulosa inicial a una comprensión clara que, por estar reflejada en un plano y unas determinaciones anexas, resulta claramente asumible y enriquecedor. Por ello, las explicaciones teóricas deben ir siempre acompañadas del correspondiente reflejo práctico. Al alumno se le proporcionan ambos contenidos.

Además, tanto de la asignatura de Legislación, como de Gestión Urbanística, el gran equipo docente que imparte estas asignaturas, ha publicado dos libros docentes "Legislación, parte general" y "Legislación y gestión urbanística en la Comunidad Valenciana", que los alumnos pueden seguir durante las clases teóricas, junto con las correspondientes presentaciones disponibles en la plataforma Poliformat, lo que ha supuesto una sólida base para el seguimiento y estudio de la lección magistral y para la posterior realización de los trabajos prácticos y proyectos. 


\subsection{Los trabajos prácticos}

Para asegurar el éxito de estas asignaturas, la metodología activa se desarrolla mediante trabajos que se plantean desde una perspectiva de supuestos reales que implican el manejo de instrumentos de planeamiento o de gestión urbanística. Se realizan en clase por grupos de alumnos bajo la supervisión del Profesor pero dando cierta autonomía al alumnado. En general resultan muy atractivos para el alumnado ya que lo introducen en el mundo real bien manejando los distintos tipos de planes o bien los recursos y sistemas para la ejecución de los mismos.

El trabajo con proyectos grupales debe ser una parte predominante en el proceso de formación del alumno en las asignaturas de contenido legal al objeto de aprender aplicando la normativa y los conocimientos adquiridos a casos reales. Además, el trabajo en pequeños grupos crea un diálogo directo entre el profesor y los alumnos que debe ser el pilar en el periodo de aprendizaje. Con ello se pretende la integración del alumno en la actividad laboral.

Por ejemplificar uno de estos trabajos, se les plantea el siguiente supuesto a trabajar por grupos reducidos de 5 alumnos en los que debe existir plena coordinación para el desarrollo del trabajo:

A cada grupo se le asigna una parcela a partir de la cual deberán realizar un proyecto que recorrerá todos los aspectos legales que deberán tener en cuenta para la finalización del mismo con el siguiente desarrollo:

1. Identificación registral y catastral de la parcela.

2. Planeamiento aplicable a la parcela: clasificación y calificación del suelo, cumplimiento o no de la condición de solar, parámetros urbanísticos aplicables, usos pormenorizados permitidos en la parcela.

3. Posibles afecciones de la parcela que puedan condicionar la obra prevista: inundabilidad, carreteras, ferrocarriles, aeropuertos, patrimonio, cauces públicos, etc.

4. Análisis, si procede, de las obras de urbanización a realizar y las cesiones pendientes al Ayuntamiento.

5. Elaboración de un breve anteproyecto y estimación de los costes de urbanización de la actuación.

6. Elaboración de planos del estado actual de la parcela, distribución de la parcela resultante, Layout e imágenes fotorealísticas.

7. Lectura y análisis de uno de los contratos a firmar entre constructora y promotora.

Durante la realización del trabajo $\mathrm{y}$, después de las explicaciones teóricas correspondientes, los alumnos trabajan en el aula con una interacción constante entre el profesorado y los grupos de alumnos resolviendo dudas y aconsejando sobre la adopción de las mejores soluciones poniendo en práctica los conocimientos legales adquiridos en las clases magistrales. 

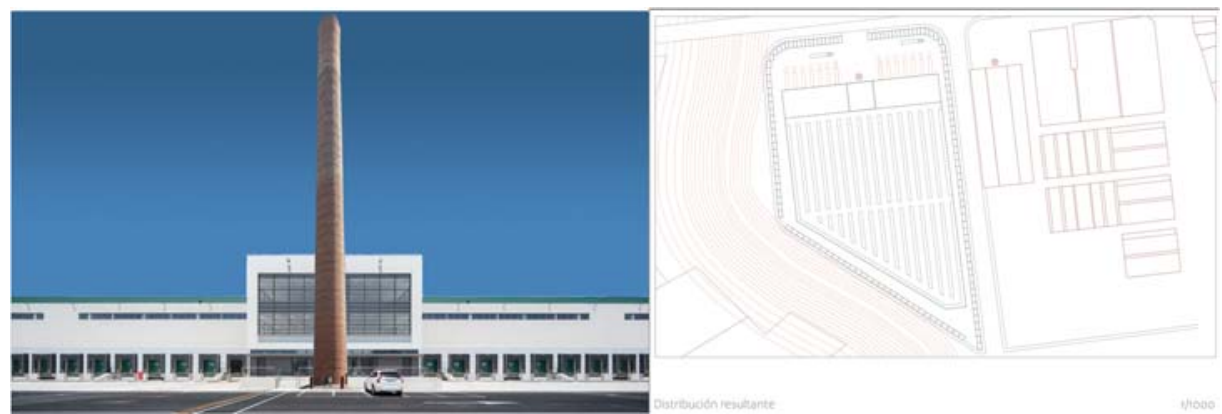

Fig. 1 Ejemplo de planos realizados por los alumnos

Otra de las actividades complementarias al trabajo grupal es, por una parte, la visita a los ayuntamientos de las parcelas asignadas al objeto de mantener una reunión con los técnicos municipales para poder recabar la información necesaria relativa a la parcela además de tener un contacto directo con la realidad del urbanismo en la administración; por otra parte, se realiza una visita guíada con un profesional junto con todos los alumnos a una de las parcelas asignadas que, en la realidad, ya está edificada para que puedan plasmar esa realidad en sus trabajos.

\section{Conclusiones}

Con esta metodología docente que se ha expuesto, realmente se han conseguido frutos muy importantes que despiertan el interés por estas asignaturas que, de otro modo, pueden resultar "ásperas" para los alumnos no acostumbrados a estudiar normativa. De este modo, los futuros arquitectos técnicos podrán aplicar la normativa correspondiente a cada una de las competencias profesionales en materia de urbanismo a través de los conocimientos adquiridos, a lo que se debe sumar la capacidad de trabajar en grupo y hablar en público con seguridad.

\section{Referencias bibliográficas}

GARCÍA ERVITI, F. et all ( 2013) " Herramientas de aprendizaje sobre regulación legal del ejercicio profesional del arquitecto". En XI Jornadas de Redes de Investigación en Docencia Universitaria. Alicante: Universidad de Alicante, $2659 \mathrm{p}$.

SANTANA RODRÍGUEZ, R.J., (2020). "El aprendizaje basado en la práctica profesional : metodologías activas". En Arquitectura v2020. Valencia: Universitat Politècnica de València. 56. 